\title{
Complete genome sequence of bacteriophage VvAW1, which infects Vibrio vulnificus
}

\author{
Olivia D. Nigro, Alexander I. Culley and Grieg F. Steward \\ University of Hawaii Department of Oceanography Center for Microbial Oceanography: \\ Research and Education Honolulu, $\mathrm{HI}$
}

Corresponding authors: Olivia Nigro (onigro@hawaii.edu), Grieg Steward (grieg@hawaii.edu)

\begin{abstract}
Investigating the bacteriophages of vibrios has led to significant insights into the evolution and pathogenicity of their host strains. This report presents the first complete genome sequence of a bacteriophage that infects the deadly human pathogen Vibrio vulnificus. The phage was isolated from the surface waters of the Ala Wai Canal, which is part of an urban watershed in eastern Honolulu, Hawai'i, USA. The phage particle is icosahedral, with a diameter of 35-40 nm, and a small non-contractile tail. The genome was sequenced in its entirety, rendering a $38 \mathrm{~kb}$ sequence located on a single, linear, circularly permuted chromosome. Here, we present the annotation and genomic features of the bacteriophage, VvAW1.
\end{abstract}

Keywords: Bacteriophage, vibriophage, Vibrio vulnificus, podophage, aquatic, virus

\section{Introduction}

Vibrio vulnificus is an opportunistic, but formidable pathogen that can cause lethal infections in humans [1]. $V$. vulnificus is responsible for upwards of $95 \%$ of shellfish-related deaths in the US, and is a common cause of lethal infections resulting from exposure of wounds to marine and brackish waters. Outbreaks of vibrio infections are often associated with flooding caused by natural disasters [2]. Bacteriophages play important, but sometimes competing, roles in the epidemiology of many bacterial pathogens, including vibrios $[3,4]$. A temperate phage enhances the virulence of $V$. harveyi, for example [5]. In the case of disease cholera the temperate phage, СТХØ, contributes to the virulence of the causative agent, $V$. cholerae, via lysogenic conversion [6], but other lytic bacteriophages appear to ameliorate cholera epidemics by lysing the pathogen [7]. Currently, little is known about phages that infect $V$. vulnificus, but they are reported to be diverse and abundant in the Pacific Northwest and Gulf Coast regions of the US [8]. V. vulnificus phages could be a major contributor to the observed variability in virulence among host strains through the processes of horizontal gene transfer and lysogenic conversion. As a first step toward evaluating these possibilities, we are undertaking the isolation and detailed characterization of model phage-host systems. We chose the Ala Wai Canal in Honolulu, HI as our sampling site, since the waters of the canal appear to have been the source of a lethal infection by Vibrio vulnificus [9]. In 2009, we isolated a bacteriophage that infects Vibrio vulnificus from the canal. Here we present a description and annotation for the complete genome sequence of Vibrio phge VvAw1.

\section{Classification and Features}

Both the Vibrio phage VvAW1, and its host $V$. vulnificus strain V93D1V were isolated from the Ala Wai Canal, a $3.1 \mathrm{~km}$ long, man-made waterway located on the southern coast of Oahu that separates Waikiki and urban Honolulu [10]. A watershed covering $42.4 \mathrm{~km}^{2}$ drains into the Ala Wai Canal, and the streams feeding the canal drain through urban areas of Honolulu, resulting in anthropogenic contamination [11]. The influx of fresh water from the streams, and the influx of seawater through the Ala Wai Harbor, creates a salinity gradient with a typical salt-wedge structure [10]. The salinity in the canal ranges from 0 to $35 \%$, depending on precipitation and tidal fluctuations (Nigro and Steward, unpublished data). Because of the local tropical climate, the temperature range of the canal is relatively narrow, usually ranging from 20 to $30^{\circ} \mathrm{C}$ (Nigro and Steward, unpublished data). The host $V$. vulnificus strain, V93D1V, is a 16S rRNA "Type A", which is not commonly associated with pathogenic infection [12]. 
The phage particle is icosahedral, with a capsid diameter of 43-45 $\mathrm{nm}$ and a tail-plus-capsid diameter of $52 \mathrm{~nm}$ (Figure 1 and Table 1). The tail appears to be short and non-contractile, which is commonly seen in members of the Podoviridae family [13]. The genome of the phage is made up of double-stranded DNA, and appeared by pulsed-field gel electrophoresis to be around $40 \mathrm{~kb}$ in length (data not shown). These structural observations, combined with phylogenetic evidence (Figure 2) have resulted in the tentative classification of VvAW1 as a member of the Podoviridae family, with an unassigned genus.

\section{Genome sequencing information Genome project history}

Vibriophage VvAW1 is the first genome sequence publicly available for a phage infecting $V$. vulnificus. As such, this sequence is a significant contribution to limited data sets of vibriophage and podovirus genomes. This genome was selected for sequencing as a first step toward better understanding the roles of bacteriophages in the ecology and virulence of $V$. vulnificus. DNA sequencing and assembly was performed at the University of Hawai'i at Mānoa, Department of Oceanography, Center for Microbial Oceanography: Research and Education (C-MORE) using the university's Advanced Studies in Genomics, Proteomics and Bioinformatics (ASGPB) sequencing facility. Genome annotation was performed both in-house as well as by submission to the IMG-ER genome annotation pipeline (Department of Energy Joint Genome Institute)
$[15,16]$. A summary of the project information is presented in Table 2.

\section{Growth conditions and DNA isolation}

Viral strain VvAW1 was isolated from waters collected at the head of the Ala Wai Canal in Honolulu, Hawai'i. On April 4, 2009, a one-liter surface water sample was collected, transported and processed within two hours of collection. Concurrent measurements of temperature and salinity were made at the time of collection. The water sample was filtered through a $0.22 \mu \mathrm{m}$ membrane filter (Sterivex; Millipore) using a peristaltic pump. The filtered Ala Wai water was then supplemented with six strains of $V$. vulnificus and incubated at room temperature overnight in order to increase the numbers of any $V$. vulnificus phages that were present through infectious replication. The water was then filtered again through a 0.22 um Sterivex filter. The presence of phages was checked by an agar overlay plaque assay against each of the six inoculated strains. Each strain was incubated separately with $0.05 \mathrm{ml}$ of the virus-containing filtrate for 10 minutes. This mixture was combined with molten soft tryptic soy agar (TSA) agar $(0.6 \%$ agar $\mathrm{w} / \mathrm{v})$ and poured onto a TSA plate. Plates were incubated at $37{ }^{\circ} \mathrm{C}$ for 72 hours, and examined for plaques every 24 hours. After 72 hours, one strain, V93DIV, had 8 plaques. A single plaque was harvested, serially diluted and plated using the soft-agar overlay technique serially, three times to ensure the isolation of a single strain of phage. Following isolation, a bacterial lawn of strain V93D1V was fully lysed to obtain a high virus titer.

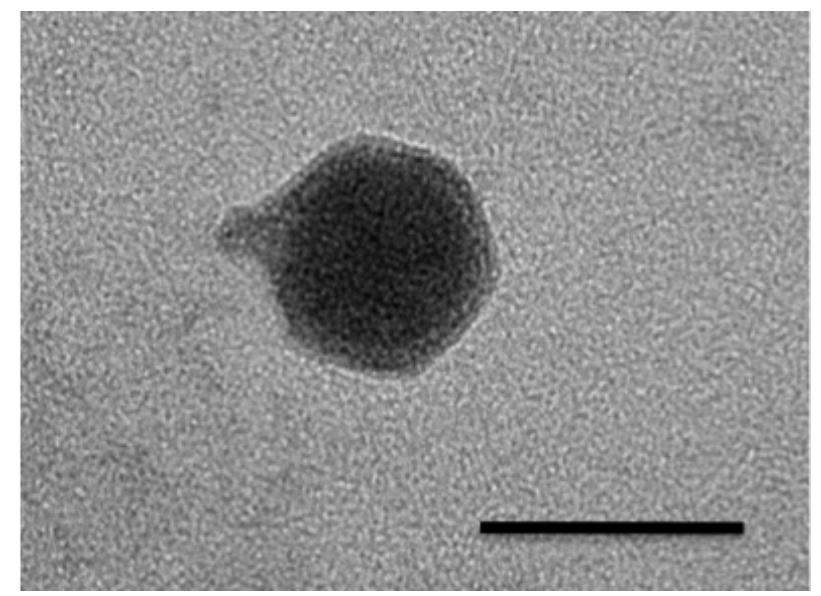

Figure 1. Transmission electron micrograph of a vibriophage VvAW1 particle. Scale bar equals $50 \mathrm{~nm}$. 


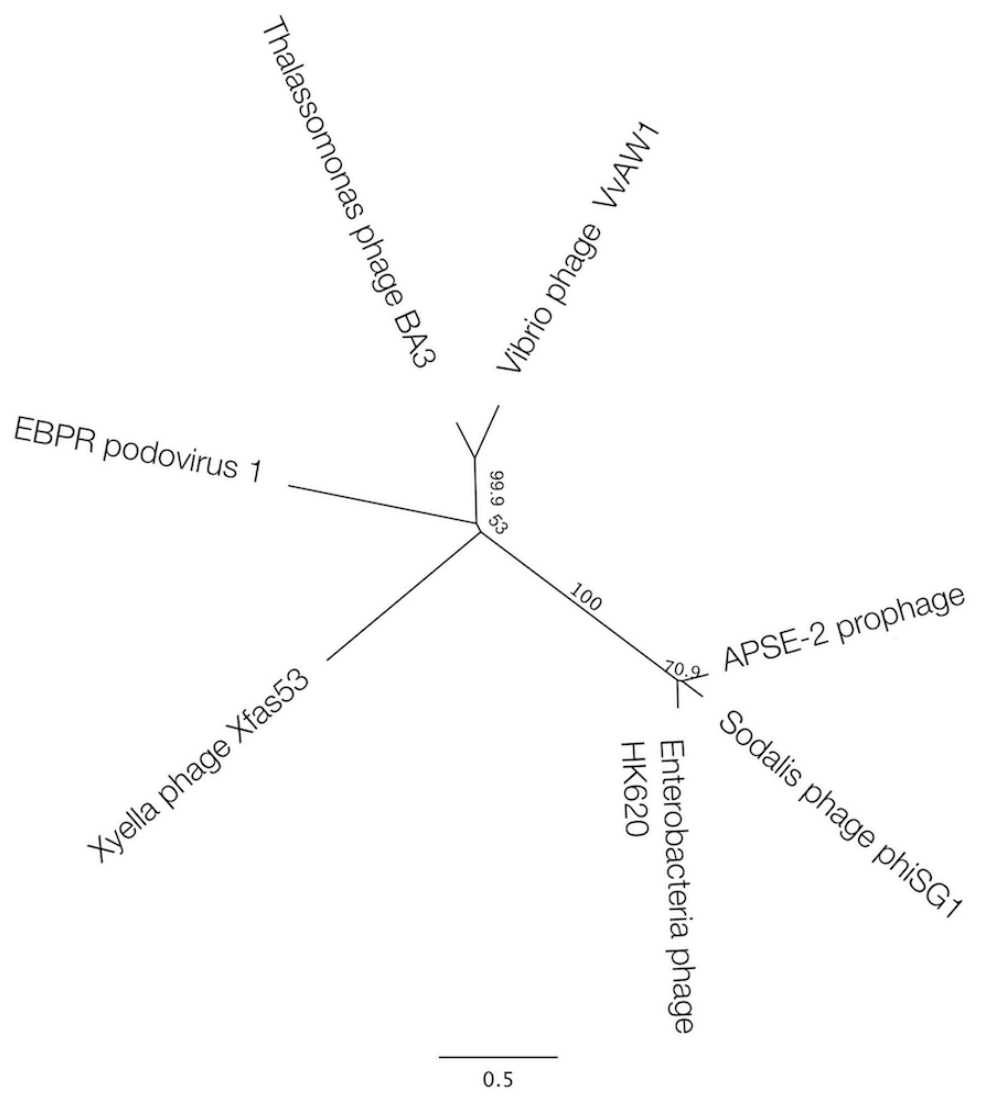

Figure 2. Unrooted maximum likelihood tree highlighting the position of Vibriophage VvAW1 relative to its most similar homologs by BLAST search (E-value $>10 \mathrm{e}-5$ ). All of the phages in the tree are classified as podoviruses, with the exception of phiSG1 and APSE-2 prophage, which are unclassified. The phages and the corresponding GenBank accession numbers for their capsid protein genes are: EBPR podovirus AEI70875; Xyella phage Xfas53, YP_003344924; Thalassomonas phage Ba3, YP_001552282; Sodalis phage phSG1, YP_516191; APSE-2 prophage, YP_002924439; and Enterobacteria phage HK620, NP_112079. These sequences were aligned using the MAFFT alignment program, version $6.814 \mathrm{~b}$, using auto settings; and the maximum likelihood tree was built using PHYML with a Whelan and Goldman substitution model, and was bootstrapped 10,000 times using the Geneious software package v 5.5.7 [14].

Viral particles were purified in a continuous equilibrium buoyant density $\mathrm{CsCl}$ gradient, spun at $29,000 \mathrm{rpm}$ for 92.5 hours at $20^{\circ} \mathrm{C}$ in an XL-80K Ultracentrifuge (Beckman) with a SW-41 rotor. CsCl was exchanged with TE buffer (10 mM Tris, 1 mM EDTA, $\mathrm{pH}$ 8) by centrifugal ultrafiltration and total nucleic acids were extracted using silicabased spin columns (QIAGEN DNeasy Blood and Tissue Kit) according to the manufacturer's instructions. Genomic DNA was hydraulically sheared to $1000-1200$ bp-sized fragments (HydroShear; GeneMachines). Size-selected DNA was end-repaired (DNATerminator End Repair Kit, Lucigen) and ligated into a pSMART HC KAN vector (Lucigen) and then transformed via electroporation into DH5alpha E. coli 10G SUPREME electrocompetent cells (Lucigen). Colonies were picked $(n=288)$ and grown in CircleGrow (MP Biomedicals) liquid media plus kanamycin and plasmid DNA was isolated. 
Table 1. Classification and general features of Vibro phage $V_{v} A W 1$

\begin{tabular}{llll}
\hline MIGS ID & Property & Term & Evidence code $^{\mathbf{a}}$ \\
& Current classification & Superkingdom: Viruses, dsDNA viruses, no RNA phase & IDA \\
& & Order Caudovirales \\
& & Family Podoviridae \\
& Genus unassigned & \\
& & Species unassigned & \\
& & & \\
MIGS-6 & Habitat & Icosahedral & IDA \\
MIGS-6.3 & Salinity & Aquatic & IDA \\
MIGS-22 & Oxygen & & \\
MIGS-15 & Biotic relationship & Obligate intracellular parasite of $V$. vulnificus & IDA \\
MIGS-14 & Pathogenicity & Infective phage of $V$. vulnificus & IDA \\
MIGS-4 & Geographic location & Ala Wai Canal, Honolulu, HI, USA & IDA \\
MIGS-5 & Sample collection time & $4 / 29 / 09,08: 40$ AM & IDA \\
MIGS-4.1 & Latitude & 21.275510 & IDA \\
MIGS-4.2 & Longitude & -157.817869 & IDA \\
MIGS-4.3 & Depth & Surface & IDA \\
MIGS-4.4 & Altitude & & \\
\hline
\end{tabular}

a) Evidence codes - IDA: Inferred from Direct Assay.

Table 2. Project information

\begin{tabular}{lll}
\hline MIGS ID & Property & Term \\
\hline MIGS-31 & Finishing quality & Closed \\
MIGS-28 & Libraries used & 1 genomic library: 800-1200 kb \\
MIGS-29 & Sequencing platforms & Sanger \\
MIGS-31.2 & Fold coverage & $8.73 \times$ \\
MIGS-30 & Assemblers & Sequencher version 4.10.1 (Gene Codes Corporation) \\
MIGS-32 & Gene calling method & GeneMark.hmm, RAST version 4.0, and IMG-ER using Prodigal \\
& Genome Database release & GenBank \\
& Genbank ID & JQ801337 \\
& Genbank Date of Release & May 31, 2012 \\
& GOLD ID & Gi14686 \\
& Project relevance & Virulence, Limited Sequence \\
\hline
\end{tabular}




\section{Genome sequencing and assembly}

The genome was sequenced using Sanger sequencing. Sequencing was performed by the Advanced Studies of Genomics, Proteomics and Bioinformatics (ASGPB) Sequencing Center at the University of Hawai'i at Mānoa (Honolulu, HI), using Applied Biosystems BigDye terminator chemistry and was run on an ABI 3730XL capillarybased DNA sequencer. The genome was assembled using Sequencher version 4.10.1 (Gene Codes Corporation). Initial reads were assembled and formed two contigs. PCR was used to close two gaps into a circular topology (in total, 6 sets of PCR primers), however restriction enzyme digestion indicated the genome of VvAW1 is linear, circularly permuted, and terminally redundant. PCR was also used to re-sequence areas with low coverage. Assembly was manually curated for errors. Final coverage of the genome is $8.7 \times$.

\section{Genome annotation}

Open reading frames (ORFs) were determined using a combination of three gene calling methods, i) the Genemark.hmm 2.0 gene prediction program [17], ii) the RAST (Rapid Annotation using Subsystem Technology) genome annotation server [18] and iii) the Integrated Microbial Genomes-Expert Review (IMG-ER) platform developed by the Joint Genome Institute, Walnut Creek, CA, USA [15]. ORFs that were identified by only one of the three methods and which showed no homology to known proteins $\left(\right.$ E-value $\left.<1 \mathrm{e}^{-5}\right)$ were not included in the annotation.
The predicted ORFs were translated and used to search the National Center for Biotechnology Information (NCBI) non-redundant database, the Conserved Domain Database (CDD), TIGRFam, Pfam, SMART, PRK, COG, and InterPro databases. The tRNAScanSE tool [19] was used to find tRNA genes. Additional manual functional annotation was performed within the IMG platform [20], within the Artemis/ACT package $[21,22]$ and using the Geneious software package v 5.5 [14]. The complete genome sequence was submitted to GenBank and assigned the accession number JQ801337.

\section{Genome properties}

The properties and the statistics of the genome are summarized in Tables 3-5.The genome has a total size of 38,682 bp of unique sequence, with one circularly permuted, terminally redundant, linear chromosome (49.1\% GC content) (Figure 3). A total of 40 genes were predicted, all of which are protein-coding genes. Of these predicted proteincoding genes, eight were assigned to a putative function three were assigned to conserved, but unknown functional categories, and the remaining were annotated as hypothetical proteins. Although only eight genes were assigned to a putative function, all but three genes showed significant sequence similarity to gene sequences, of either known or unknown function, in the NCBI database (Table 5). No paralogs were identified in this genome.

Table 3. Nucleotide content and gene count levels of the genome

\begin{tabular}{lrr}
\hline Attribute & Value & \% $^{\text {of }}$ total $^{\mathbf{a}}$ \\
\hline Size (bp) & 38,682 & 100 \\
G+C content (bp) & 18,998 & 49.11 \\
Coding region (bp) & 37,543 & 97.1 \\
Total genes & 40 & 100 \\
RNA genes & 0 & 0 \\
Protein-coding genes & 40 & 100 \\
Genes in paralog clusters & 0 & 0 \\
Genes assigned to COGs & 12 & 30 \\
1 or more conserved domains & & \\
2 or more conserved domains & & \\
3 or more conserved domains & & \\
4 or more conserved domains & & \\
Genes with signal peptides & 6 & 5 \\
Genes with transmembrane helices & 2 & 0 \\
Paralogous groups & 0 & \\
\hline
\end{tabular}

a) The total is based on either the size of the genome in base pairs or the total number of protein coding genes in the annotated genome. 
Table 4. Number of genes associated with the 25 general COG functional categories

\begin{tabular}{|c|c|c|c|}
\hline Code & Value & $\%$ age $^{\mathrm{a}}$ & Description \\
\hline$J$ & 0 & 0 & Translation \\
\hline A & 0 & 0 & RNA processing and modification \\
\hline K & 2 & 5 & Transcription \\
\hline $\mathrm{L}$ & 3 & 7.5 & Replication, recombination and repair \\
\hline B & 0 & 0 & Chromatin structure and dynamics \\
\hline $\mathrm{D}$ & 0 & 0 & Cell cycle control, mitosis and meiosis \\
\hline Y & 0 & 0 & Nuclear structure \\
\hline V & 0 & 0 & Defense mechanisms \\
\hline $\mathrm{T}$ & 2 & 5 & Signal transduction mechanisms \\
\hline M & 1 & 2.5 & Cell wall/membrane biogenesis \\
\hline $\mathrm{N}$ & 1 & 2.5 & Cell motility \\
\hline Z & 0 & 0 & Cytoskeleton \\
\hline W & 0 & 0 & Extracellular structures \\
\hline$U$ & 0 & 0 & Intracellular trafficking and secretion \\
\hline $\mathrm{O}$ & 0 & 0 & Posttranslational modification, protein turnover, chaperones \\
\hline $\mathrm{C}$ & 1 & 2.5 & Energy production and conversion \\
\hline G & 0 & 0 & Carbohydrate transport and metabolism \\
\hline $\mathrm{E}$ & 0 & 0 & Amino acid transport and metabolism \\
\hline $\mathrm{F}$ & 0 & 0 & Nucleotide transport and metabolism \\
\hline $\mathrm{H}$ & 0 & 0 & Coenzyme transport and metabolism \\
\hline I & 0 & 0 & Lipid transport and metabolism \\
\hline$P$ & 0 & 0 & Inorganic ion transport and metabolism \\
\hline Q & 0 & 0 & Secondary metabolites biosynthesis, transport and catabolism \\
\hline $\mathrm{R}$ & 1 & 2.5 & General function prediction only \\
\hline S & 3 & 7.5 & Function unknown \\
\hline- & 28 & 70 & Not in COGs \\
\hline
\end{tabular}

a) The total is based on the total number of protein coding genes in the annotated genome.

\section{Insights from the genome sequence Comparative genomics}

Significant similarity was observed between Vibriophage VvAW1 and Ba3, which infects the coral pathogen Thalassomonas loyana, both in terms of gene order and gene homology (Figures 3 and 4). Bacteriophage genomes have been described as mosaic, with areas of intense similarity amalgamated with areas that appear to be unrelated [23]. The genome of VvAW1 displays extensive mosaicism, with some regions closely related to $\mathrm{Ba} 3$, and some apparently unrelated. Many of the areas of the genome that do not show homology to $\mathrm{Ba} 3$, show significant similarity to another phage, Pseudomonas phage F116 (Figure 4). Only one of the predicted VvAW1genes (gene 13) showed significant sequence similarity to both Ba3 and F116. Mosaic patterns in bacteriophage genomes support the theory that horizontal gene transfer plays a role in phage evolution [23]. 
Nigro et al.

Table 5. Vibriophage VvAW1 gene annotations*.

\begin{tabular}{|c|c|c|c|c|c|c|}
\hline Gene & Strand & Function or Similarity & Evidence or Organism & Accession Num. & $\%$ Id & E-Value \\
\hline 1 & - & DNA methylase & COG4646 & COG4646 & 24 & $8.0 \mathrm{e}^{-}$ \\
\hline 2 & - & hypothetical protein & Thalassomonas phage Ba3 & NC_009990 & 43 & $2.0 \mathrm{e}^{\ldots}$ \\
\hline 3 & - & phage DNA transfer protein & Xyella fastidiosa M12 & СР000941 & 35 & $1.0 \mathrm{e}^{19}$ \\
\hline 4 & - & hypothetical phage protein & PHA00672 & cl10253 & $\mathrm{nr}$ & $2.4 \mathrm{e}^{-}$ \\
\hline 5 & - & hypothetical protein & None & $\mathrm{n} / \mathrm{a}$ & $\mathrm{n} / \mathrm{a}$ & $\mathrm{n} / \mathrm{a}$ \\
\hline 6 & - & phage tail collar protein & pfam07484 & $\mathrm{n} / \mathrm{a}$ & $\mathrm{nr}$ & $1.5 \mathrm{e}^{-15}$ \\
\hline 7 & - & phage stabilization prot., gp 10 & Xyella phage Xfas53 & GQ421471 & 36 & $1.0 \mathrm{e}^{25}$ \\
\hline 8 & - & hypothetical protein & Thalassomonas phage Ba3 & NC_009990 & 34 & $2.0 \mathrm{e}^{-5}$ \\
\hline 9 & - & hypothetical protein & Thalassomonas phage Ba3 & NC_009990 & 52 & $4.0 \mathrm{e}^{m}$ \\
\hline 10 & - & P22 coat protein, gp5 & pfam11651 & cl0373 & $\mathrm{nr}$ & $1.3 \mathrm{e}^{-2}$ \\
\hline 11 & - & scaffold protein & EBPR podovirus & AE170876 & 36 & $4.0 \mathrm{e}^{\infty}$ \\
\hline 12 & - & portal protein & EBPR podovirus & AE170876 & 38 & $1.0 \mathrm{e}^{\mathrm{n} z}$ \\
\hline 13 & - & terminase large subunit & Shigella phage Shfl1 & NC_015456 & 39 & $0.0 \mathrm{e}^{-\infty}$ \\
\hline 14 & - & terminase, small subunit & pfam03592 & cl01513 & $\mathrm{nr}$ & $4.0 \mathrm{e}^{m}$ \\
\hline 15 & - & hypothetical phage protein & PHA000821 & cl10282 & $\mathrm{nr}$ & $5.8 \mathrm{e}^{10}$ \\
\hline 16 & - & holin protein & $\begin{array}{l}\text { Psychrobacter phage } \\
\text { psymv2 }\end{array}$ & AEO01029 & 46 & $1.0 \mathrm{e}^{\prime \prime}$ \\
\hline 17 & - & hypothetical protein & Acinetobacter phage AB1 & ADO14413 & 48 & $4.0 \mathrm{e}^{2}$ \\
\hline 18 & - & hypothetical protein & Vibrio sp. AND4 & ZP_02196938 & 32 & $3.0 \mathrm{e}^{12}$ \\
\hline 19 & - & endolysin & Bacteriophage P27 & 103807.1 & 37 & $2.0 \mathrm{e}^{\prime \prime}$ \\
\hline 20 & - & hypothetical protein & none & $\mathrm{n} / \mathrm{a}$ & $\mathrm{n} / \mathrm{a}$ & $\mathrm{n} / \mathrm{a}$ \\
\hline 21 & - & ssDNA binding protein & COG0629 & COG0629 & 43 & $4.0 \mathrm{e}^{\cdots}$ \\
\hline 22 & - & hypothetical protein & none & $\mathrm{n} / \mathrm{a}$ & $\mathrm{n} / \mathrm{a}$ & $\mathrm{n} / \mathrm{a}$ \\
\hline 23 & - & con. protein, unknown func. & DUF2303 & cl02338 & $\mathrm{nr}$ & $5.1 \mathrm{e}^{\ldots+}$ \\
\hline 24 & - & hypothetical protein & CPS-53 prophage & AEQ15784 & 47 & $3.0 \mathrm{e}$ \\
\hline 25 & - & hypothetical protein & Pseudomonas phage D3 & NP_061574 & 34 & $3.0 \mathrm{e}^{19}$ \\
\hline 26 & - & RNA-binding regulator protein & COG1551 & COG1551 & 43 & $6.0 \mathrm{e}^{-10}$ \\
\hline 27 & - & exonuclease & pfam00929 & cl0219 & $\mathrm{nr}$ & $1.1 \mathrm{e}^{17}$ \\
\hline 28 & - & hypothetical protein & Pseudomonas phage F116 & AY625898 & 40 & $0.0 \mathrm{e}^{-\infty}$ \\
\hline 29 & - & hypothetical protein & Pseudomonas phage F116 & AY625898 & 43 & $0.0 \mathrm{e}^{-\infty}$ \\
\hline 30 & - & hypothetical protein & Pectobacteria phage ZF40 & AFC22460.1 & 35 & $4.0 \mathrm{e}^{-15}$ \\
\hline 31 & - & hypothetical protein & none & $\mathrm{n} / \mathrm{a}$ & $\mathrm{n} / \mathrm{a}$ & $\mathrm{n} / \mathrm{a}$ \\
\hline 32 & + & lambda-like repressor, HTH mot. & SSF47413 & IPR010982 & $\mathrm{nr}$ & $1.6 \mathrm{e}$ \\
\hline 33 & + & hypothetical protein & Thalassomonas phage Ba3 & NC_009990 & 46 & $3.0 \mathrm{e}^{19}$ \\
\hline 34 & + & con. protein, unknown func. & DUF3268 & cl13172 & $\mathrm{nr}$ & $1.7 \mathrm{e}^{\infty}$ \\
\hline 35 & + & hypothetical protein & Vibrio cholerae MZO-3 & ZP_01955042 & 40 & $1.0 \mathrm{e}$ \\
\hline 36 & + & hypothetical protein & Opitutacecae sp. TAV5 & ZP_09596846 & 50 & $1.0 \mathrm{e}^{2}$ \\
\hline 37 & + & con. protein, unknown func. & DUF1367 & cl06231 & $\mathrm{nr}$ & $1.7 \mathrm{e}^{s i}$ \\
\hline 38 & + & con. protein, unknown func. & DUF1364 & cl06229 & $\mathrm{nr}$ & $5.1 \mathrm{e}^{*}$ \\
\hline 39 & - & hypothetical protein & Roseobacter denitrificans & YP_771820 & 29 & $4.0 \mathrm{e}^{m}$ \\
\hline 40 & - & cellulosome enzyme & Acinetobacter baumanni & ZP_05829744 & 36 & $1.0 \mathrm{e}^{\mathrm{m}}$ \\
\hline
\end{tabular}

*Genes are listed by number, along with their predicted function, if known, followed by the nature of the evidence (e.g., COG group) that supports the functional classification. Genes with no functional prediction, but with significant $\left(\mathrm{E}<10^{-5}\right)$ sequence similarity to genes in the $\mathrm{NCBI}$ database as determined by blastp, are listed, followed by the name of the organism in which the similar gene was found. 


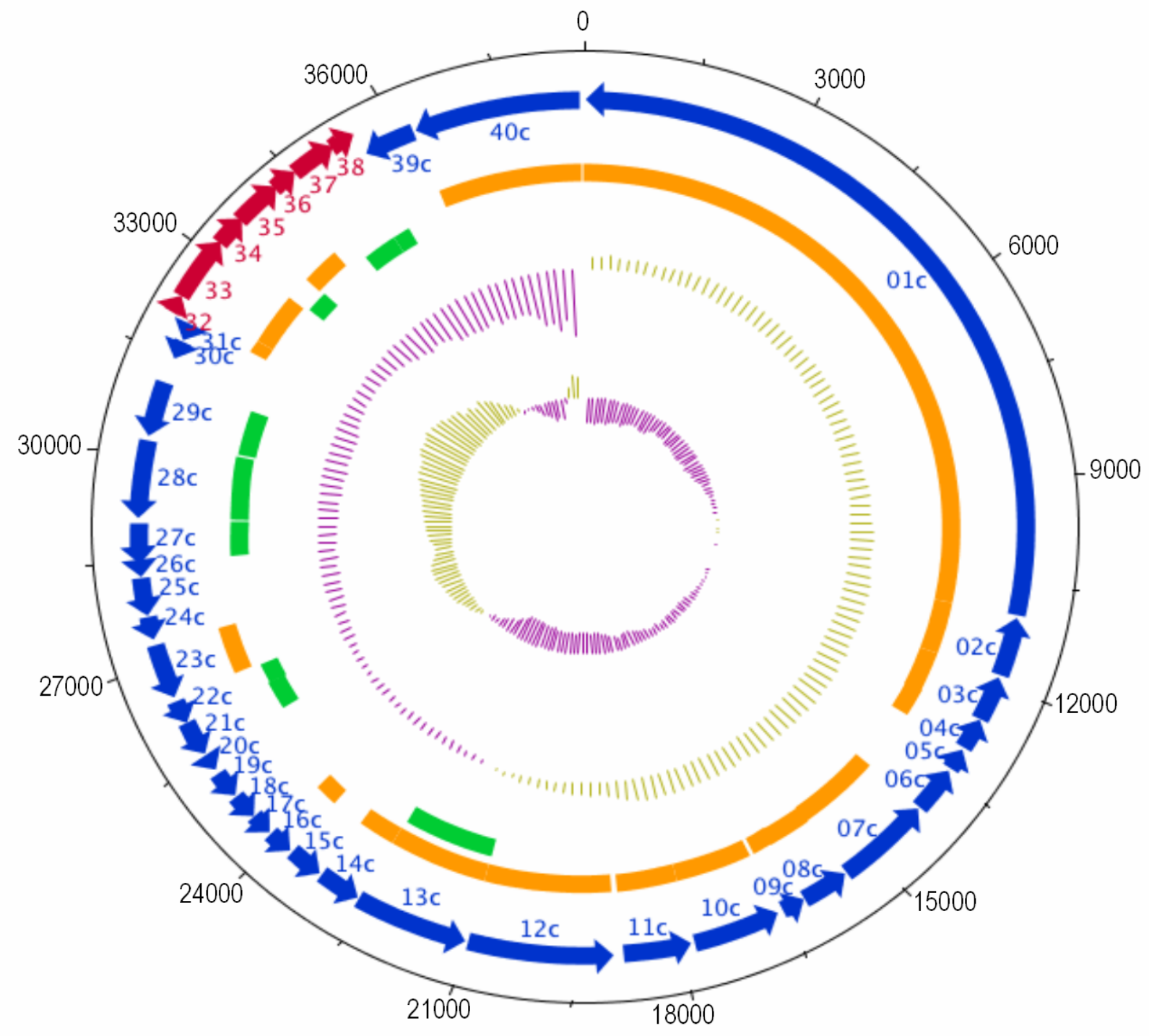

Figure 3. Genome map of $\mathrm{V}_{\mathrm{VAW}}$ 1. The outer two tracks show numbered predicted genes and direction of transcription (red: forward direction, blue: reverse). The orange and green tracks indicate genes with significant sequence similarity $\left(>10 \mathrm{e}^{-5}\right.$ ) to Thalassomonas phage Ba3, and Pseudomonas phage F116, respectively. Moving inward, the tracks show the \%GC content (purple=low \%GC) and GC skew $([\mathrm{G}-\mathrm{C}] /[\mathrm{G}+\mathrm{C}]$ ) (innermost) of the genome. 


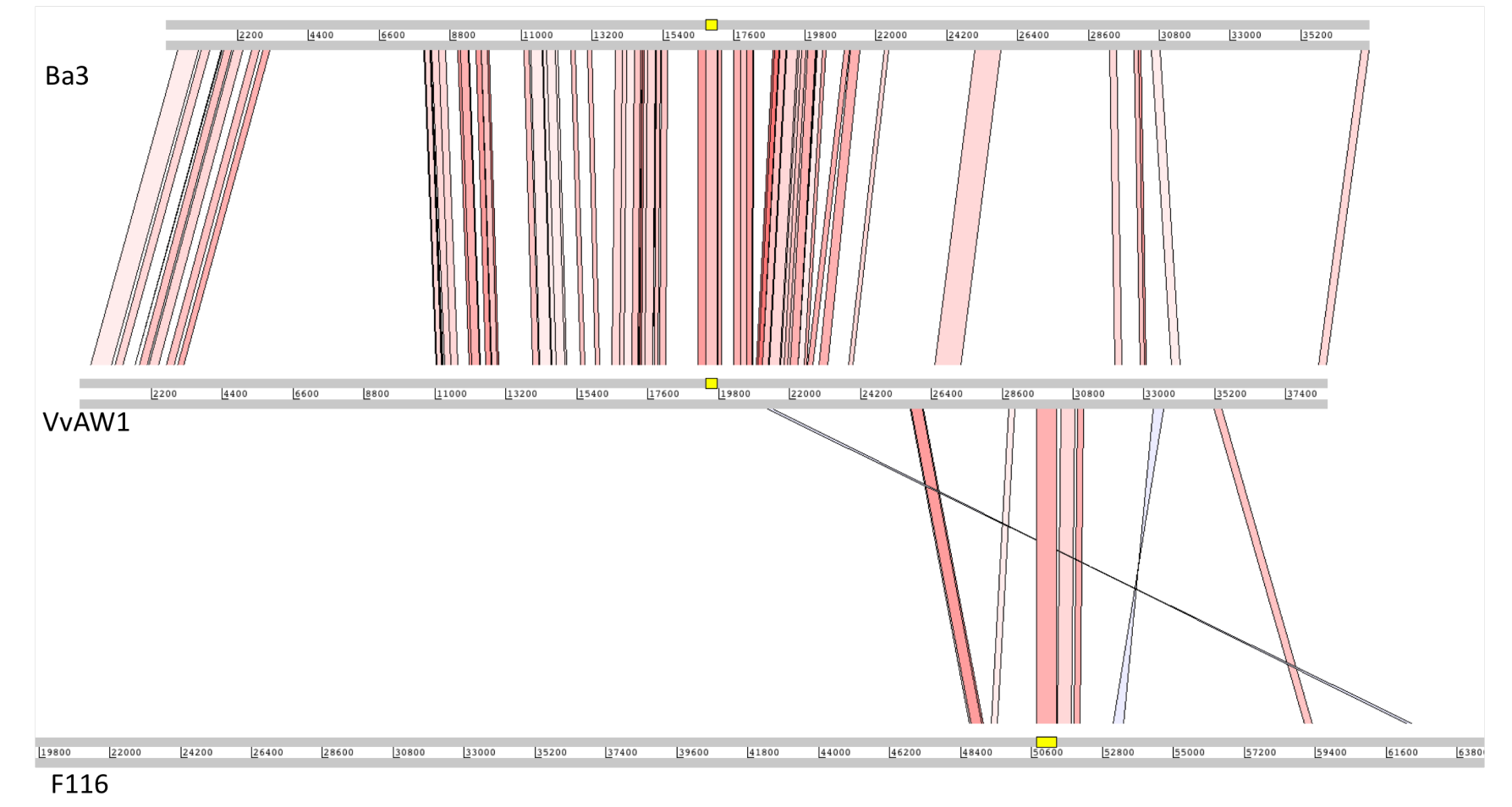

Figure 4. Whole genome comparison of Vibrio phage VvAW1 to Thalassomonas phage Ba3, and Pseudomonas phage F116. This figure was generated using the Artemis Comparison Tool (ACT) [21]. Genomes were aligned using WebACT, using default tblastx settings, with E-value set to $10 \mathrm{e}^{-4}$, and genetic code set to eubacterial. ACT display was set to show homologous regions with BLAST scores $>40$, and sequence similarity $>25 \%$.

\section{VvAW1 replication strategy}

The life cycle and replication strategy of Vibrio phage VvAW1 have not been determined, however while propagating the phage it was found that infected cultures did not completely clear, and plaques were turbid (data not shown), suggesting that the bacteriophage is temperate [24]. Analysis of the genome sequence further supports the hypothesis that the phage is temperate. As determined by homology searches using the CDD, Pfam motif analysis, and InterProScan, the predicted protein product of gene 32 is a transcriptional regulator with homology to the Enterobacteria phage lambda (lambda) repressor C1, which is responsible for maintaining lysogeny in E. coli. The VvAW1 C1 homolog also displays a helix-turn-helix motif. The putative $\mathrm{C} 1$ repressor gene is a location of transcriptional divergence in the VvAW1 genome, similar to lambda (Figure 5a). The temperate bacteriophage lambda has a central regulatory circuit that has been wellstudied. Divergently transcribed repressors (C1 and Cro) regulate passage into the lytic or lysogenic cycle [27]. Although sequence homology was not seen in gene product 31 to the Cro repressor, genome arrangement between the two phages is conserved (Figure 5a). The intergenic space between the $\mathrm{C} 1$ and Cro genes in lambda is the site of two key promoters involved in regulatory events. Although the promoters were not identified in this region of the VvAW1 genome, GC content in the intergenic spacer is low (37\%) relative to the VvAW1 genome.

Synteny between VvAW1 and lambda persists in other regions of the genome. The lytic pathway of lambda, up-regulated by Cro production, leads to the transcription of "early" and "late" phage genes. These genes are located downstream of the Cro repressor. The early genes, which encode proteins involved in DNA replication, are transcribed first, followed by the morphogenetic or late genes, which encode phage assembly proteins. This modular organization of the genome is typical of tailed bacteriophages [28]. Temperate phages show a striking conservation of gene order with regard to their morphogenetic genes with very few exceptions to the clustering and specific order of these genes [26]. The genome of VvAW1 shows the gene clustering of function and conservation of gene order of early and late genes that is 
characteristic of temperate phages (Figure 5b). Notably, the VvAW1 genome is missing the "tail shaft" and "tape measure" genes, as is the case for the genome of the Salmonella phage P22. The absence of these genes in P22 can be attributed to the fact that P22 is a podophage, and therefore has a short tail. The absence of these genes in VvAW1 as well, corroborates the morphological and genomic evidence and further supports the inclusion of this phage in the family Podoviridae.

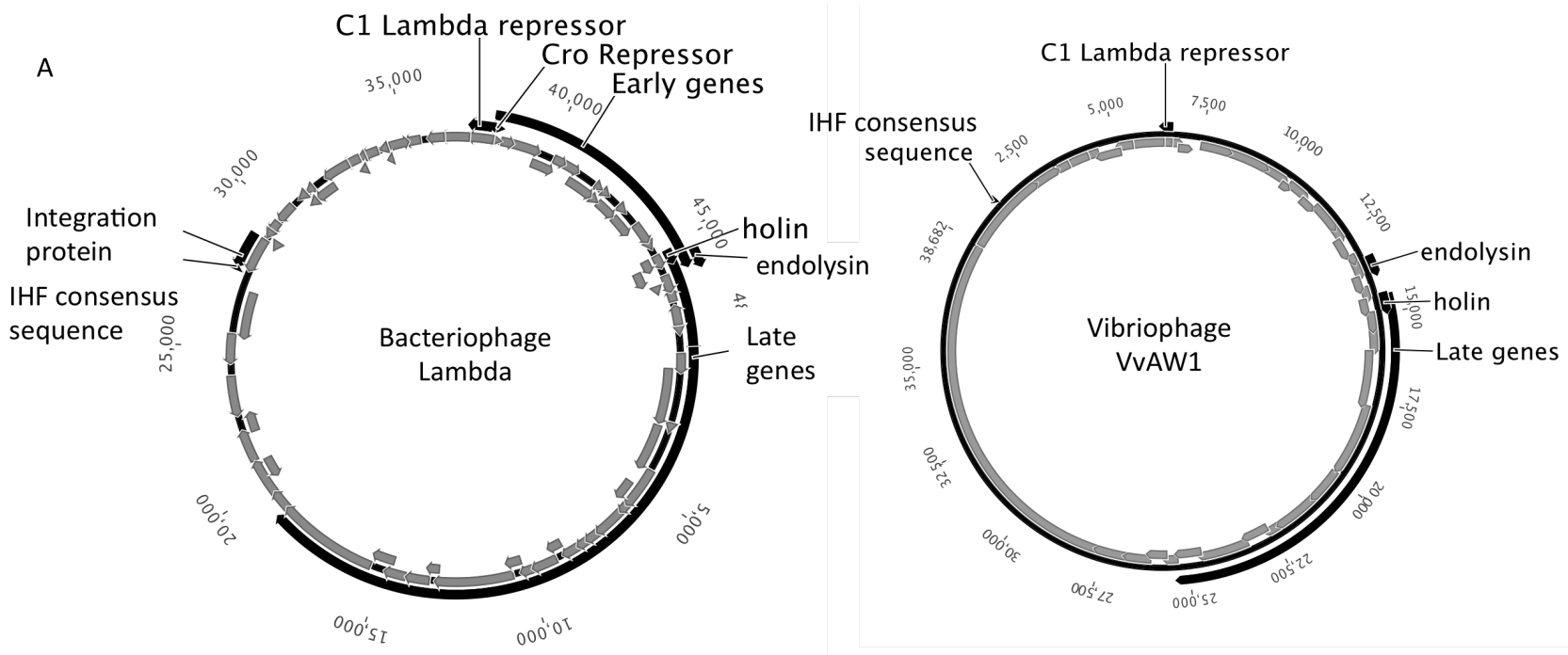

B

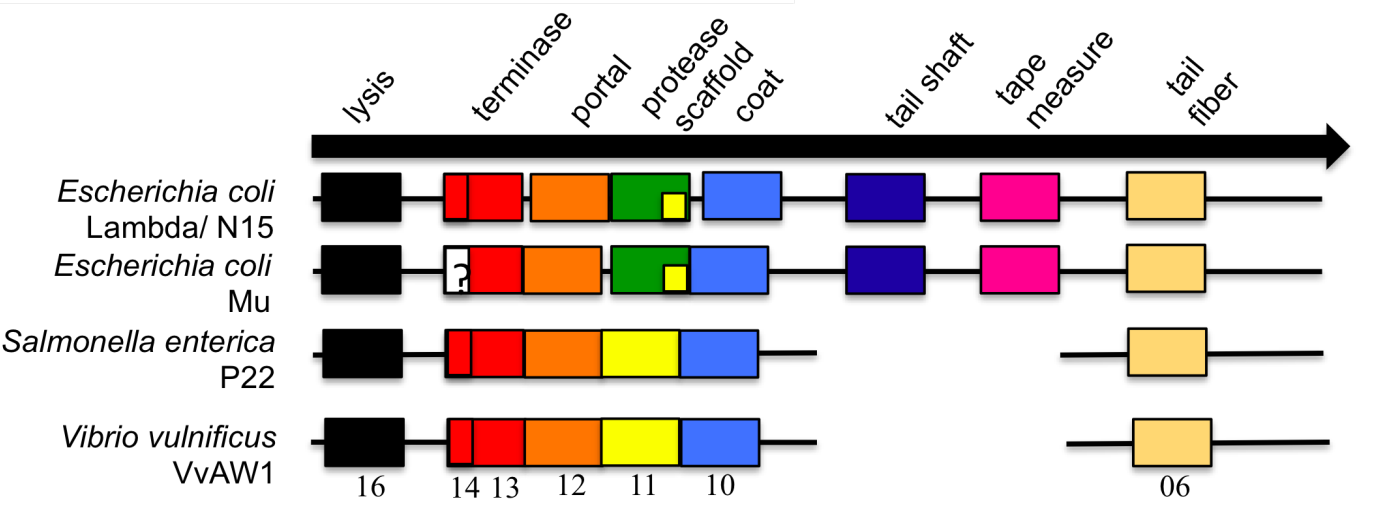

Figure 5. Comparison of similar regions of the lambda, and Vibriophage VvAW1 genomes. (A) Circularized genomes of lambda and VvAW1 highlighting synteny between the two phages. The genome of phage lambda was accessed from GenBank, accession number NC_001416 [25]. The genome maps were drawn using the Geneious software package [14].(B) Late genes of temperate phages. This figure was redrawn and modified from Casjens, 2003 [26]. Similar colors represent genes with conserved order, but not necessarily sequence similarity. Spaces between the colored squares indicate that additional genes lie between the indicated genes. The numbers below the VvAW1 genome indicate gene number as described in Table 5.

We were unable to identify an integrase gene in the genome of VvAW1. Integrase genes regulate the integration of viral genomes into the genome of their host, and in lambda this gene is located downstream of the $\mathrm{C} 1$ lambda repressor (Figure $5 A)[25,29]$. The integrase gene may be present and not sufficiently similar to other integrases to be identified by sequence similarity. It is also possible that VvAW1 replicates as a plasmid, which has been observed in F116, as well as Vibriophage VHS1 [30,31]. Immediately downstream of the intergrase gene in the lambda genome is the attP site, which contains integration host factor (IHF) binding sites. We have identified a region in VvAW1 that has the IHF binding consensus sequence AWWTCAANNNNTR downstream of the putative lambda-like repressor [32]. 
Nigro et al.

The consensus sequence lies within gene 40 in VvAW1. Gene 40 does not show homology to other integrase genes. Blastp analysis of gene 40 indicated homology to the dockerin type I cellulosome protein of several bacterial species. If the identified IHF sequence is part of the attP site of VvAW1, gene 40 could be of bacterial origin, as a result of genetic recombination.

\section{Conclusion}

According to our analysis of the Vibriophage VvAW1 genome, this phage is most likely a member of the viral family Podoviridae. The

\section{Acknowledgments}

This work was supported by grants from NSF (OCE 08-26650, ANT-0944851) and the University of Hawaii Sea Grant College Program (NA090AR4171048), and funding from the NSF-

\section{References}

1. Dziuban EJ, Liang JL, Craun GF, Hill V, Yu PA, Painter J, Moore MR, Calderon RL, Roy SL, Beach MJ. Surveillance for waterborne disease and outbreaks associated with recreational waterUnited States, 2003-2004. MMWR Surveill Summ 2006; 55:1-30. PubMed

2. Alajo SO, Nakavuma J, Erume J. Cholera in endemic districts in Uganda during El Niño rains: 2002-2003. Afr Health Sci 2006; 6:93-97. PubMed

3. Cerveny KE, Depaola A, Duckworth DH, Gulig PA. Phage therapy of local and systemic disease caused by Vibrio vulnificus in iron-dextran-treated mice. Infect Immun 2002; 70:6251-6262. PubMed http://dx.doi.org/10.1128/IAI.70.11.6251$\underline{6262.2002}$

4. Brüssow H, Canchaya C, Hardt WD. Phages and the Evolution of Bacterial Pathogens: from Genomic Rearrangements to Lysogenic Conversion. Microbiol Mol Biol Rev 2004; 68:560602. PubMed http://dx.doi.org/10.1128/MMBR.68.3.560$\underline{602.2004}$

5. Khemayan K, Prachumwat A, Sonthayanon B, Intaraprasong A, Sriurairatana S, Flegel TW. The complete genome sequence of a virulenceenhancing siphophage VHS1 from Vibrio harveyi. Appl Environ Microbiol 2012; 78:2790-2796. PubMed http://dx.doi.org/10.1128/AEM.05929-11

6. Waldor MK, Mekalanos J. Lysogenic conversion by a filamentous phage encoding cholera toxin. genome shows modular organization and mosaicism. Portions of the genome show synteny with the genome of bacteriophage lambda. High sequence similarity was observed between VvAW1 and the Thalassomonas phage Ba3, as well as the Pseudomonas phage F116. Functional predictions of VvAW1 genes indicate the possibility of a lysogenic replication strategy, however an integrase gene could not be identified in the genome. It is possible that VvAW1 lysogenizes its host, without integrating into the host genome, replicating as a plasmid.

supported Center for Microbial Oceanography: Research and Education (EF 04-24599). We would like to thank Chris Schvarcz for assistance with TEM imaging.

Science 1996; 272:1910-1914. PubMed http://dx.doi.org/10.1126/science.272.5270.1910

7. Faruque SM, Naser IB, Islam MJ, Faruque ASG, Ghosh AN, Nair GB, Sack DA, Mekalanos JJ. Seasonal epidemics of cholera inversely correlate with the prevalence of environmental cholera phages. Proc Natl Acad Sci USA 2005; 102:17021707. PubMed http://dx.doi.org/10.1073/pnas.0408992102

8. DePaola A, Motes M, Chan A, Suttle C. Phages infecting Vibrio vulnificus are abundant and diverse in oysters (Crassostrea virginica) collected from the Gulf of Mexico. Appl Environ Microbiol 1998; 64:346-351. PubMed

9. Creamer B, Moreno L. "Horrible, horrible death" by infection. Honolulu Advertiser. April 8, 2006.

10. Heinen De Carlo E, Anthony S. Spatial and temporal variability of trace element concentrations in an urban subtropical watershed, Honolulu, Hawaii. Appl Geochem 2002; 17:475492. http://dx.doi.org/10.1016/S08832927(01)00114-7

11. Laws EA, Doliente D, Hiayama J, Hokama ML, Kim K, Li D, Minami S, Morales C.

Hypereutrophication of the Ala Wai Canal, Oahu, Hawaii: prospects for cleanup. Pac Sci 1993; 47:59-75.

12. Kim YR, Lee SE, Kim CM, Kim SY, Shin EK, Shin $\mathrm{DH}$, Chung SS, Choy HE, Progulske-Fox A, Hillman JD. Characterization and pathogenic 
significance of Vibrio vulnificus antigens preferentially expressed in septicemic patients. Infect Immun 2003; 71:5461-5471. PubMed http://dx.doi.org/10.1128/IAI.71.10.5461$\underline{5471.2003}$

13. King $A M Q$, Adams MJ, Carstens EB. Lefkowitz EJ eds. Virus Taxonomy. 9th ed. (King AMQ, Adams MJ, Carstens EB, Lefkowitz EJ, eds.). Boston: Elsevier Academic Press; 2012.

14. Drummond A, Ashton B, Buxton S. Geneious v5.4, Available from http://www.geneious.com. 2011.

15. Markowitz VM, Mavromatis K, Ivanova NN, Chen IMA, Chu K, Kyrpides NC. IMG ER: a system for microbial genome annotation expert review and curation. Bioinformatics 2009; 25:2271-2278. PubMed http://dx.doi.org/10.1093/bioinformatics/btp393

16. Integrated Microbial Genomes-Expert Review. http://img.jgi.doe.gov

17. Besemer J, Borodovsky M. Heuristic approach to deriving models for gene finding. Nucleic Acids Res 1999; 27:3911-3920. PubMed http://dx.doi.org/10.1093/nar/27.19.3911

18. Aziz RK, Bartels D, Best AA, Dejongh $M$, Disz $T$, Edwards RA, Formsma K, Gerdes S, Glass EM, Kubal M. The RAST Server: Rapid Annotations using Subsystems Technology. BMC Genomics 2008; 9:75. PubMed http://dx.doi.org/10.1186/1471-2164-9-75

19. Lowe TM, Eddy SR. tRNAscan-SE: a program for improved detection of transfer RNA genes in genomic sequence. Nucleic acids research 1997;25:0955-0964.

20. Markowitz VM, Szeto E, Palaniappan K, Grechkin Y, Chu K, Chen IM, Dubchak I, Anderson I, Lykidis A, Mavromatis K. The integrated microbial genomes (IMG) system in 2007: data content and analysis tool extensions. Nucleic Acids Res 2008; 36:D528-D533. PubMed http://dx.doi.org/10.1093/nar/gkm846

21. Carver TJ, Rutherford KM, Berriman $M$, Rajandream MA, Barrell BG, Parkhill J. ACT: the Artemis comparison tool. Bioinformatics 2005; 21:3422-3423. PubMed http://dx.doi.org/10.1093/bioinformatics/bti553

22. Rutherford K, Parkhill J, Crook J, Horsnell T, Rice P, Rajandream MA, Barrell B. Artemis: sequence visualization and annotation. Bioinformatics 2000; 16:944-945. PubMed http://dx.doi.org/10.1093/bioinformatics/16.10.944
23. Hendrix RW, Smith M, Burns RN, Ford ME, Hatfull GF. Evolutionary relationships among diverse bacteriophages and prophages: all the world's a phage. Proc Natl Acad Sci USA 1999; 96:21922197. PubMed http://dx.doi.org/10.1073/pnas.96.5.2192

24. Barksdale L, Arden SB. Persisting bacteriophage infections, lysogeny, and phage conversions. Annu Rev Microbiol 1974; 28:265-299. PubMed http://dx.doi.org/10.1146/annurev.mi.28.100174.0 $\underline{01405}$

25. Sanger F, Coulson AR, Hong GF, Hill DF, Petersen GB. Nucleotide sequence of bacteriophage lambda DNA. J Mol Biol 1982; 162:729-773. PubMed http://dx.doi.org/10.1016/0022-2836(82)90546-0

26. Casjens S. Prophages and bacterial genomics: what have we learned so far? Mol Microbiol 2003;

49:277-300. PubMed http://dx.doi.org/10.1046/j.13652958.2003.03580.x

27. Schubert RA, Dodd IB, Egan JB, Shearwin KE. Cro's role in the $\mathrm{Cl}$-Cro bistable switch is critical for lambda's transition from lysogeny to lytic development. Genes Dev 2007; 21:2461-2472. PubMed http://dx.doi.org/10.1101/gad.1584907

28. Krupovic M, Prangishvili D, Hendrix RW, Bamford $\mathrm{DH}$. Genomics of Bacterial and Archaeal Viruses: Dynamics within the Prokaryotic Virosphere. Microbiol Mol Biol Rev 2011; 75:610-635. PubMed http://dx.doi.org/10.1128/MMBR.00011$\underline{11}$

29. Kikuchi Y, Nash HA. The bacteriophage lambda int gene product. A filter assay for genetic recombination, purification of int, and specific binding to DNA. I Biol Chem 1978; 253:71497157. PubMed

30. Byrne M, Kropinski AM. The genome of the Pseudomonas aeruginosa generalized transducing bacteriophage F116. Gene 2005; 346:187-194. PubMed http://dx.doi.org/10.1016/j.gene.2004.11.001

31. Khemayan K, Pasharawipas T, Puiprom O, Sriurairatana S, Suthienkul O, Flegel TW. Unstable Lysogeny and Pseudolysogeny in Vibrio harveyi Siphovirus-Like Phage 1. Appl Environ Microbiol 2006; 72:1355-1363. PubMed http://dx.doi.org/10.1128/AEM.72.2.1355$\underline{1363.2006}$

32. Goodman SD, Velten NJ, Gao Q, Robinson S, Segall AM. In vitro selection of integration host factor binding sites. J Bacteriol 1999; 181:32463255. PubMed 